of the most important factors which heretofore have produced confusion in scientific names. The date, Jan. 1, 1931, was selected (instead of making the amendment immediately effective) in order to give authors ample opportunity to accommodate themselves to the new rule.

The Commission unanimously adopted the following resolution :

(a) It is requested that an author who publishes a name as new shall definitely state that it is new, that this be stated in only one (i.e. in the first) publication, and that the date of publication be not added to the name in its first publication.

(b) It is requested that an author who quotes a generic name, or a specific name, or a subspecific name, shall add at least once the author and year of publication of the quoted name or a full bibliographic reference.

The foregoing resolution was adopted in order to inhibit the confusion which has frequently resulted from the fact that authors have occasionally published a given name as ' new' in two to five or more different articles of different dates - up to five years in exceptional cases.

The three propositions submitted by Dr. Franz Poche, of Vienna, failed to receive the necessary number of votes in Commission to permit of their being recommended to the Congress. Out of a possible 18 votes for each proposition, Poche's proposition I. received 9 votes, II. received 6 votes, and III. received 7 votes.

Zoological, medical, and veterinary journals throughout the world are requested to give to the foregoing the widest possible publicity in order to avoid confusion and misunderstanding.

C. W. Stiles,

Secretary to Commission.

United States Public Health Service, Washington, D.C.

\section{Salivary Secretions of Blood-sucking Insects in Relation to Blood Goagulation.}

Most blood-sucking insects at the time of biting inject into their host an irritant which causes a skin reaction, generally a wheal, more or less conspicuous and itching in relation to the habituation of the host to the particular parasite. Although these injections are of such great importance in the transmission of disease, their purpose has in no case been properly elucidated hitherto. Macloskie forty years ago suggested that the salivary secretion of mosquitoes prevents the blood from clotting on the way to the stomach, but the work of Nuttall and Shipley (1903), and of Schaudinn (1904), threw doubt on this theory. Cornwall and Patton (1914) proved the presence of an anticoagulin in the salivary glands of several bloodsucking insects and ticks, and also showed that a neutralising coagulant enzyme sometimes existed in the stomach.

Dr. H. M. O. Lester, of this Tsetse Investigation, has found that the tsetse-flies, Glossina, have both an anticoagulin in the salivary secretion and a coagulin in the mesenteron, and, after studying the behaviour of these enzymes in vitro, has come to the conclusion that their influence on the coagulation of blood is similar to that of antikinase and kinase respectively. The anticoagulin thus differs from the anticoagulant enzyme of the leech, Hirudin, which is said to be an anti-thrombin.

We have proved that Macloskie's theory, if applied to the tsetse-fly, is correct. The entire salivary glands of $G$. tachinoides may be removed by a very simple operation without killing the fly and often without causing appreciable shock. Flies from which the glands are thus removed draw blood normally for a time, causing no wheal on the most susceptible skin, and may survive for long. There is never any regeneration of the glands. One fly lived for 58 days, taking 26 meals of human blood and producing 4 healthy larvæ, while others survived to 14 days. Sooner or later the flies choke, or sometimes get convulsions, and can no longer draw blood. It is then found that the lumen of the proboscis and the œesophagus are occluded by clot, and generally the capacious crop is also full of firmly clotted blood. In the case of the tsetse-fly, therefore, the injection of salivary secretion into the host is not a necessary preliminary to feeding but rather in the nature of an accident, because the secretion mixes with the indrawn blood at the very tip of the proboscis, so that a certain amount inevitably escapes into the tissues of the host.

The amount of secretion mixed with the blood is enough to delay the coagulation of mammalian blood for 2-3 hours at least, but the coagulin of the mesenteron is so powerful that in a matter of seconds it has neutralised the anticoagulin and formed a small clot at the posterior end of the meal. The main function of this clot appears to be that it puts a brake on to the fluid meal and holds it in the proper region of the gut while digestion begins. LL. Lloyd.

The Tsetse Investigation,

$$
\text { Azare, via Bauchi, }
$$

Nigeria, N.P.

Nov. 16.

\section{The Thermal State of the Earth's Grust.}

The product of the rate of increase of temperature with depth in the earth's crust into the thermal conductivity of the surface rocks gives the rate of loss of heat from the interior. When allowance is made for the residual effects of the original heat, this gives a most important datum concerning the rate of generation of heat below, and hence, if the radioactivity of the rocks is known, to an estimate of the thickness of the radioactive layer. Hitherto it has been good enough, in discussions of this problem, to adopt mean values of the temperature gradient, the conductivity, and the radioactivity. It has now, however, become worth while to allow for variation of conductivity with depth, and to attend more to details in the vertical distribution of radioactive matter. When this is done as well as is at present possible, the agreement of the results with those obtained from the study of near earthquakes is practically perfect (Gerlands Beiträge z. Geophysik, 18, 1-29; 1927).

The next step towards understanding the earth's thermal state is to find out why the observed temperature gradient varies from place to place; the mean for North America, for example, is substantially less than for Europe. This may be attributed to a different thickness of the radioactive layers, or to different percentages of radioactive matter in them. But these questions cannot be effectively discussed without an additional datum that has not yet attracted. the attention of observers, namely, the conductivity of the uppermost layer (usually sedimentary). The actual temperature gradients are measured in this layer, and we cannot find the rate of conduction of heat to the surface without a knowledge of its conductivity. Conductivities of sedimentary rocks are given in some of the standard physical tables, but these refer to dried specimens, and the effective conductivity of the same rocks in situ is probably considerably higher on account of the part played in transferring heat by water in the interstices. Further, there is no known reason why it should not vary from place to place.

No. 3036, VoL. 121] 